\title{
Editorial: The Birth of Endocrine Pathology
}

\author{
Kalman Kovacs • Sylvia L. Asa
}

Published online: 9 May 2013

(C) Springer Science+Business Media New York 2013

During the last 50 years, phenomenal progress was achieved in the better understanding of a large number of diseases including those of the endocrine glands. Previously unknown hormones were discovered; new methods were developed to measure hormone levels in blood, body fluids, and tissues; and new information emerged relating to the functions of hormones and their regulation. Research focused on animal experiments moved from observational to interventional as the ability to engineer animal models developed. With progress towards personalized care, the investigation of primary human samples became the basis for targeted therapies and precision medicine; newly introduced imaging methods and substantial advances in surgical techniques assumed fundamental importance.

The role of pathology increases as novel therapies require more advanced and precise understanding of human diseases. The need for subspecialization and multidisciplinary care teams has led to greater demand for publications that can integrate knowledge in specific areas of medicine. We foresaw a need more than 25 years ago when endocrine pathology was as yet an unrecognized discipline. There were many journals that published papers on clinical endocrinology and therapeutic approaches in endocrine diseases; there were pathology journals that were interested in disease pathogenesis and some that focused on the pathology of tumors of several organs. However, there was no journal focusing on the pathology of diseases of the endocrine glands. Indeed, much of endocrine pathology was lost in the realm of other pathology interests:

K. Kovacs $(\square)$

Department of Laboratory Medicine, Divsion of Pathology,

St. Michael's Hospital, 30 Bond Street,

Toronto, ON M5B1W8, Canada

e-mail: kovacsk@smh.ca

\section{S. L. Asa}

University Health Network, 200 Elizabeth St. Rm 11E-051,

Toronto, ON M5G 2C4, Canada

e-mail: Sylvia.Asa@uhn.ca pituitary pathology was the territory of neuropathologists, thyroid and parathyroid diseases were handled by head-and-neck pathologists, adrenals and the gonads were examined by genitourinary pathologists, and the large number of neuroendocrine pathologies of the gastroenteropancreatic system fell under the auspices of gastrointestinal pathologists. The commonality of hormone function and regulation, the structural essence of endocrine cells, and the systemic impact of endocrine disorders led us to propose the need for an overarching approach; the drive to work with endocrinologists, endocrine surgeons, and endocrine oncologists was the impetus for our work. We decided to challenge the existing order, to define a new discipline, and to create a new journal: Endocrine Pathology.

The first issue was published in March 1990. As the two founding editors, we were honored to have as successors Drs. Ricardo V. Lloyd, Arthur S. Tischler, Virginia A. Livolsi, and Anne Marie McNicol who have continued to bring our dream to reality. Their efforts have created a journal that is recognized and respected internationally. The impact factor of the journal continues to rise as the scope and quality of the publications increase. We are grateful to the excellent editorial board, the reviewers, the publishing companies, and all the authors who have chosen to publish their work in Endocrine Pathology. We are optimistic that the journal will continue to be successful and will inspire readers to pursue deeper insights into the pathology of endocrine diseases.

William Osler said: "Everywhere the old order changes, and happy they who can change with it." John F. Kennedy said: "Change is the law of life. And those who look only to the past or present are certain to miss the future." We are constantly aware of the need for change and the importance of providing our discipline with the tools to ensure its sustainability. We hope that the legacy of this journal and of the discipline of endocrine pathology will be one of insight, foresight, and excellence. For indeed, as Alan Kay said, "the best way to predict the future is to invent it." 